\title{
Activation of FXR promotes intestinal metaplasia of gastric cells via SHP-dependent upregulation of the expression of CDX2
}

\author{
HAINING ZHOU ${ }^{1,2^{*}}$, ZHEN NI $^{1 *}$, TING LI $^{1}$, LINNA SU $^{1}$, LIANFENG ZHANG $^{2}$, NA LIU $^{1}$ and YONGQUAN SHI ${ }^{1}$ \\ ${ }^{1}$ State Key Laboratory of Cancer Biology and Institute of Digestive Diseases, Xijing Hospital, \\ Fourth Military Medical University, Xi'an, Shaanxi 710032; 2 Department of Gastroenterology, \\ The First Affiliated Hospital of Zhengzhou University, Zhengzhou, Henan 450052, P.R. China
}

Received April 13, 2017; Accepted January 29, 2018

DOI: $10.3892 / \mathrm{ol} .2018 .8342$

\begin{abstract}
Gastric intestinal metaplasia (IM) induced by bile acid is a precancerous lesion of gastric adenocarcinoma and is associated with the expression of caudal-related homeobox 2 (CDX2). In the present study, the role of farnesoid X receptor $(\mathrm{FXR})$ on the regulation of $\mathrm{CDX} 2$ in gastric cells was investigated and the underlying molecular mechanisms were examined. Human gastric cell lines were treated with chenodeoxycholic acid (CDCA) or FXR agonist GW4064. Cells were treated with CDCA in the presence or absence of the FXR antagonist or FXR siRNA transfection. Next, cells were treated with CDCA in the presence or absence of SHP siRNA transfection and FXR, CDX2 and SHP mRNA and protein levels were determined by reverse transcription-quantitative polymerase chain reaction and western blot analysis. A chromatin immunoprecipitation assay was performed to examine the relationship between FXR and SHP and the expressions of FXR and CDX2 in gastritis and IM tissues were detected using immunohistochemistry. The results revealed that CDCA was able to induce CDX2 expression, which could be blocked by inhibition or knockdown of FXR. Mechanistically, FXR directly induced the expression of small heterodimer partner (SHP). SHP knockdown significantly decreased CDCA-induced CDX2 expression. ChIP results
\end{abstract}

Correspondence to: Professor Yongquan Shi, State Key Laboratory of Cancer Biology and Institute of Digestive Diseases, Xijing Hospital, Fourth Military Medical University, 169 Changlexi Road, Xi'an, Shaanxi 710032, P.R. China

E-mail: shiyquan@fmmu.edu.cn

"Contributed equally

Abbreviations: IM, intestinal metaplasia; CDX2, caudal-related homeobox 2; FXR, farnesoid X receptor; SHP, small heterodimer partner; CDCA, chenodeoxycholic acid; EGFR, epidermal growth factor receptor; NF-kB, nuclear factor-kB

Key words: bile acid, intestinal metaplasia, caudal-related homeobox 2 , farnesoid $\mathrm{X}$ receptor, small heterodimer partner indicated that FXR could directly bind SHP promoter and promote SHP expression. Finally, immunohistochemistry results demonstrated that the expression levels of CDX2 and FXR in human IM lesions were significantly higher, compared with those in gastritis lesions, and were positively correlated. Collectively, these results revealed that the activation of FXR and sequential direct transcriptional induction of SHP were involved in the expression of CDX2 induced by bile acid in gastric IM lesions.

\section{Introduction}

Intestinal metaplasia (IM) induced by bile acid is considered to be the most important precancerous lesion of intestinal type gastric cancer (1). IM is characterized by the expression of caudal-related homeobox 2 (CDX2), a transcription factor that is important for intestinal tract development and differentiation during embryogenesis $(2,3)$. In adult humans, CDX2 acts as a tumor suppressor, which is strictly confined to the intestine and cannot be detected in normal gastric mucosa. However, the aberrant expression of CDX2 in the upper digestive tract is pro-oncogenic, and is observed in gastric IM and gastric carcinoma (4). It has been reported that transgenic mice overexpressing CDX2 caused extensive IM progression in the stomach, which may be associated with the downstream intestinal specific genes of CDX2 (5). Therefore, CDX2 is considered to be a trigger in the progression of gastric IM.

Farnesoid X receptor (FXR) is a member of nuclear metabolic receptors. FXR also functions as a transcription factor and directly binds to DNA as a heterodimer with common partner retinoid $X$ receptor to regulate the expression of downstream genes when activated by bile acid. It has been demonstrated that FXR is involved in the bile acid-induced progression of IM and the expression of various intestinal specific genes, including CDX2 (6,7). However, the molecular mechanism remains to be fully elucidated.

In the present study, it was found that FXR and CDX2 were upregulated and positively correlated in gastric IM tissues. In addition, the results demonstrated that the molecular mechanism by which the expression of CDX2 is mediated involved the direct transcriptional regulation of small heterodimer partner (SHP) by the nuclear receptor FXR. 


\section{Materials and methods}

Tissue specimens and immunohistochemistry. Biopsy specimens of gastric IM (61 patients) and gastritis (55 patients) were obtained under endoscopy following the provision of informed consent from patients at the Department of Pathology of Xijing Hospital (Xi'an, China) between March 2011 and February 2013. Corresponding clinical data were obtained from medical records. The present study was approved by the Institutional Review Board of Xijing Hospital.

The tissues were fixed in $10 \%$ formalin and paraffin-embedded. IM and gastritis were diagnosed through standard hematoxylin and eosin staining. For immunohistochemistry, tissue sections $(4 \mu \mathrm{m})$ were incubated with rabbit polyclonal anti-CDX2 antibody (cat. no. sc-134468; Santa Cruz Biotechnology, Inc., Dallas, TX, USA; dilution 1:100) and anti-FXR antibody (cat. no. sc-13063, Santa Cruz Biotechnology, Inc.; dilution 1:100) at $4^{\circ} \mathrm{C}$ overnight. Negative controls were incubated with rabbit IgG (Dako, Glostrup, Denmark). The intensity of staining was divided into four grades (intensity scores): Negative (0), weak (1), moderate (2) and strong (3). The histological score was determined using the following formula: Overall score=percentage score $\mathrm{x}$ intensity score. An overall score of 0-12 was calculated and graded as negative (score 0-2), or positive (score 3-12).

Cell culture and treatment. The normal GES-1 human gastric epithelial cell line, AGS, BGC-823 and MKN-45 human gastric adenocarcinoma cell lines, and SGC-7901 human gastric carcinoma cell line were obtained from the Academy of Military Medical Science (Beijing, China) and cultured in RPMI-1640 medium. All cells were maintained at $37^{\circ} \mathrm{C}$ in a humidified incubator containing 5\% $\mathrm{CO}_{2}$. Chenodeoxycholic acid (CDCA; Sigma-Aldrich; Merck Millipore, Darmstadt, Germany; cat. no. c9377), GW4064 (FXR agonist; Tocris Bioscience, Bristol, UK; cat. no, 2473), and the FXR antagonist guggulsterone (Gug; Sigma-Aldrich; Merck Millipore; cat. no. 1302214) were dissolved in dimethyl sulfoxide. The cells $\left(1-5 \times 10^{5}\right)$ were seeded in six-well and $6 \mathrm{~cm}$ cell culture dish respectively. When the cells reached $80-90 \%$ confluence, they were serum-deprived overnight and then treated with CDCA $(0,50,100$ and $200 \mu \mathrm{M})$ in the presence or absence of Gug $(5,50$ and $100 \mu \mathrm{M})$ or GW4064 $(0.25,0.5,1,5$ and $10 \mu \mathrm{M})$ for $24 \mathrm{~h}$ under $37^{\circ} \mathrm{C}$.

Plasmid construction and transfection. The small interfering RNA (siRNA) oligonucleotides for FXR, SHP and the negative control were designed and synthesized by Guangzhou RiboBio Co., Ltd. (Guangzhou, China). The sequences were as follows: FXR siRNA: Sense: 5'-GCCACUUCUUGAUGUGCUATT-3', anti-sense: 5'-UAGCACAUCAAGAAGUGGCTT-3', SHP siRNA: Sense: 5'-GCAGUGGCUUCAAUGCUGUTT-3' anti-sense: 5'-ACAGCAUUGAAGCCACUGCTT-3'. Transient transfection was performed using DharmaFect Transfection reagent (Thermo Fisher Scientific, Inc., Waltham, MA, USA; cat. no. T-2002-03) according to the manufacturer's protocols. The cells were collected $48 \mathrm{~h}$ following transfection.

RNA extraction and reverse transcription-quantitative polymerase chain reaction ( $R T-q P C R)$ analysis. Total RNA from the cultured cells was extracted using TRIzol (Invitrogen;
Thermo Fisher Scientific, Inc.) according to the manufacturer's protocol, and cDNA was synthesized using the PrimeScript RT reagent kit (Takara Biotechnology Co., Ltd., Dalian, China; cat. no. DRR037A). qPCR was performed using SYBR Premix Ex Taq II (Takara Biotechnology Co., Ltd.; cat. no. DRR820A) and measured in a LightCycler 480 system (Roche Diagnostics, Basel, Switzerland). The thermocycling procedure was as follows: Initial denaturation at $95^{\circ} \mathrm{C}$ for $5 \mathrm{~min}$, followed by 39 cycles at $95^{\circ} \mathrm{C}$ for $5 \mathrm{sec}$, and at $60^{\circ} \mathrm{C}$ for $30 \mathrm{sec}$. GAPDH was used as the endogenous control and relative quantification was evaluated using the $2^{-\Delta \Delta C q}$ method (8). The primers are listed in Table I.

Western blot analysis. Proteins were prepared from the cultured cells with complete cell lysis with protease and phosphatase inhibitor (Roche Diagnostics). Protein concentrations were determined using bicinchoninic acid Protein Assay kit (Thermo Fisher Scientific, Inc.; cat. no. 23227). The denatured proteins $(10-50 \mu \mathrm{g})$ were separated on SDS-PAGE (10\% gel) and transferred onto nitrocellulose filter membranes. The membranes were blocked in $10 \%$ skimmed milk in TBST $(0.1 \%$ Tween-20) for $1 \mathrm{~h}$ at room temperature and then incubated with the following primary antibodies: Rabbit polyclonal CDX2 (Cell Signaling Technology, Inc.; cat. no. 12306; 1:1,000), rabbit polyclonal kruppel like factor 4 (KLF4; Cell Signaling Technology, Inc.; cat. no. 12173; 1:1,000), rabbit polyclonal villin-1 (Cell Signaling Technology, Inc.; cat. no. $2369 ; 1: 1,000)$, rabbit polyclonal mucin 2 (MUC2; Abcam, Cambridge, MA, USA; cat. no. ab76774; 1:1,000), rabbit polyclonal FXR (Abcam; cat. no. ab155124; 1:1,000), rabbit polyclonal SHP (Santa Cruz Biotechnology, Inc.; cat. no. sc-2305; $1: 1,000)$, mouse monoclonal $\beta$-actin (Sigma-Aldrich; Merck Millipore; cat. no. A2228; 1:2,000) at $4^{\circ} \mathrm{C}$ overnight. Subsequent to be washed with TBST for 3 time, membranes were incubated with anti-Immunoglobulin G conjugated with horseradish peroxidase antibody (Cell Signaling Technology, Inc.; cat. no. 8457/3700; 1:2,000) at room temperature for $1 \mathrm{~h}$. Then the bands were scanned using the ChemiDocXRS+ imaging system (Bio-Rad Laboratories, Inc., Hercules, CA, USA) and quantified using Quantity One v4.6 software (Bio-Rad Laboratories, Inc.).

Chromatin immunoprecipitation (ChIP) assay. The GES-1 cells were prepared for the ChIP assay using a ChIP assay kit (Thermo-Fisher Scientific, Inc.; cat. no. 26156) according to the manufacturer's protocols. Immunoprecipitation was performed with rabbit polyclonal anti-FXR antibody (Abcam; cat. no. ab28676; $10 \mu \mathrm{g} / \mathrm{ml}$ ) and normal rabbit IgG (Abcam; cat. no. ab172730; $4 \mu \mathrm{g} / \mathrm{ml}$ ) as a control at $4^{\circ} \mathrm{C}$ overnight. The resulting precipitated DNA specimens were analyzed using PCR ( $25 \mu 1$, total reaction system with $2 \mu$ DNA) to amplify a 159-bp region of the SHP promoter with the following primers: Forward, 5'-GGCTGGCTTCCTGGCTTAGC-3' and reverse 5'-CTGCCCCTTATCAGATGACTC-3'. The thermocycling procedure was as follows: Initial denaturation at $98^{\circ} \mathrm{C}$ for $5 \mathrm{~min}$, followed by 33 cycles at $98^{\circ} \mathrm{C}$ for $30 \mathrm{sec}, 58^{\circ} \mathrm{C}$ for $20 \mathrm{sec}$ and at $68^{\circ} \mathrm{C}$ for $20 \mathrm{sec}$. The PCR products were resolved electrophoretically on a $3 \%$ agarose gel and stained with ethidium bromide. The gel was subjected to Peiqing JS-380A auto-focus gel image analyzing system (P\&Q Science \& Technology Inc. Shanghai, China, http://www.peiqing.com/.). 
Table I. Primers used for reverse transcription-quantitative polymerase chain reaction analysis.

\begin{tabular}{lll}
\hline Gene & \multicolumn{1}{c}{ Forward primer $\left(5^{\prime}-3^{\prime}\right)$} & \multicolumn{1}{c}{ Reverse primer $\left(5^{\prime}-3^{\prime}\right)$} \\
\hline CDX2 & GCTGGAGCTGGAGAAGGAGTT & CCTTTGCTCTGCGGTTCTG \\
MUC2 & CAACCAGCACGTCATCCTGAA & GATGCAAATGCTGGCATCAAAG \\
KLF4 & AAGAGTTCCCATCTCAAGGCAA & GGGCGAATTTCCATCCACAG \\
Villin1 & GCTTGGCAACTCTAGGGACTGG & TGAGGTTGCTGTTAGCATTGACAC \\
SHP & CAAGGAATATGCCTGCCTGA & TTCCAGGACTTCACACAGCAC \\
GAPDH & ATGTCGTGGAGTCTACTGGC & TGACCTTGCCCACAGCCTTG
\end{tabular}

CDX2, caudal-related homeobox 2; MUC2, mucin 2; KLF4, kruppel like factor 4; SHP, small heterodimer partner.

Statistical analysis. SPSS software (version 12.0; SPSS Inc., Chicago, IL, USA) was used for statistical analyses. Continuous data are presented as the mean \pm standard error of the mean. Differences between two groups were analyzed by an unpaired two-tailed Student's t-test and differences between multiple groups were analyzed by one-way analysis of variance with Tukey's post-hoc test. Categorical variables are presented as rate and were compared between two groups with a $\chi^{2}$ test. The linear correlation coefficient (Pearson's R) was calculated to determine the correlation between the expression of FXR and CDX2 in tissues. $\mathrm{P}<0.05$ was considered to indicate a statistically significant difference.

\section{Results}

CDCA-induced expression of CDX2 and its downstream intestinal-specific genes MUC2, KLF4 and villin-1 in GES-1 cells. To investigate the possible effects of bile acid on the progression of gastric IM, the present study investigated the expression of CDX2 and its downstream genes, MUC2, KLF4 and villin-1, in the GES-1 normal human gastric epithelial cell line following treatment with CDCA. As shown in Fig. 1A, when the GES-1 cells were treated with CDCA $(0,50,100$ and $200 \mu \mathrm{mol} / \mathrm{l}$ ) for $12 \mathrm{~h}$, the expression of endogenous CDX2 and downstream MUC2, KLF4 and villin-1 increased at the mRNA levels in a dose-dependent manner. Compared with the control group, the highest mRNA expression levels of CDX2, MUC2, KLF4 and villin-1 were observed in the $200 \mu \mathrm{mol} / 1$ group $(\mathrm{P}<0.01)$. Significantly increased protein expression levels of CDX2, MUC2 and villin-1 were also observed, as measured using western blot analysis, in the $200 \mu \mathrm{mol} / 1$ CDCA-treated GES-1 cells.

CDCA increases the expression of CDX2 via the activation of FXR. FXR is a member of the nuclear hormone receptor superfamily and is involved in the homeostasis of bile acid, cholesterol and lipid. Previous studies have reported that the expression of FXR is confined to the liver, intestine, kidney and adrenal glands (9). To investigate whether the activation of FXR is involved in the CDCA-induced expression of CDX2, the present study aimed to identify the baseline expression pattern of FXR in several human gastric epithelial cells and gastric cancer cells. As shown in Fig. 2, FXR was expressed in the normal human gastric epithelial cell and human gastric cancer cell lines. Of note, the expression level was significantly lower in the GES-1 cells, compared with that in the other gastric cancer cells.

Subsequently, the expression of CDX2 was detected following treatment of the GES-1 and BGC-823 cells with the FXR agonist (GW4064). The results of the western blot analysis showed that the expression of CDX2 was significantly upregulated by different concentrations of GW4064 (1, 5 and $10 \mu \mathrm{mol} / \mathrm{l}$; Fig. 3A). Gug is reported to compete for FXR ligand-binding domain with CDCA and to be an antagonist ligand for FXR (10). Accordingly, GES-1 and BGC-823 cells were treated with CDCA in the absence or presence of different concentrations of Gug. As shown in Fig. 3B, the effect of the promotion of CDCA on the expression of CDX2 was eliminated by Gug in a dose-dependent manner.

To further investigate the role of FXR in the upregulation of CDX2, the siRNA of FXR was transfected into GES-1 cells. As shown in the Fig. 3C, the FXR-siRNA significantly reduced the protein level of FXR, and the knockdown of FXR eliminated the protein expression of CDX2 induced by CDCA.

SHP is required for the FXR-induced expression of CDX2. Extensive evidence suggests that the nuclear receptor SHP is a classic downstream gene of FXR in bile acid biosynthesis. SHP is reported to be required for the DCA-induced expression of CDX1 (11). Therefore, it was hypothesized that CDCA-FXR may induce the expression of CDX2 through SHP. First, to examine whether CDCA-FXR induces the expression of SHP in gastric cells, the expression of SHP was measured in GES-1 cells following CDCA treatment with or without GW4064 and Gug. The mRNA and protein levels of SHP were induced by CDCA and were considerably enhanced by GW4064 treatment. The upregulation of the CDCA-induced protein expression of SHP was eliminated by Gug (Fig. 4A). The knockdown of SHP by siRNA resulted in a significant reduction in the CDCA-induced expression of CDX2 (Fig. 4B).

In order to further confirm whether the increase in the upregulation of SHP as a result of the expression of FXR is the consequence of direct DNA-binding activity, a ChIP assay was designed using the GES-1 cell line. As expected, the DNAs from the chromatin sample binding with FXR antibody contained SHP mRNA, and increased binding to the SHP promoter site was observed following CDCA treatment (Fig. 4C). Taken together, CDCA-FXR regulated the expression of SHP by binding to its promoter site in gastric cells. 
A
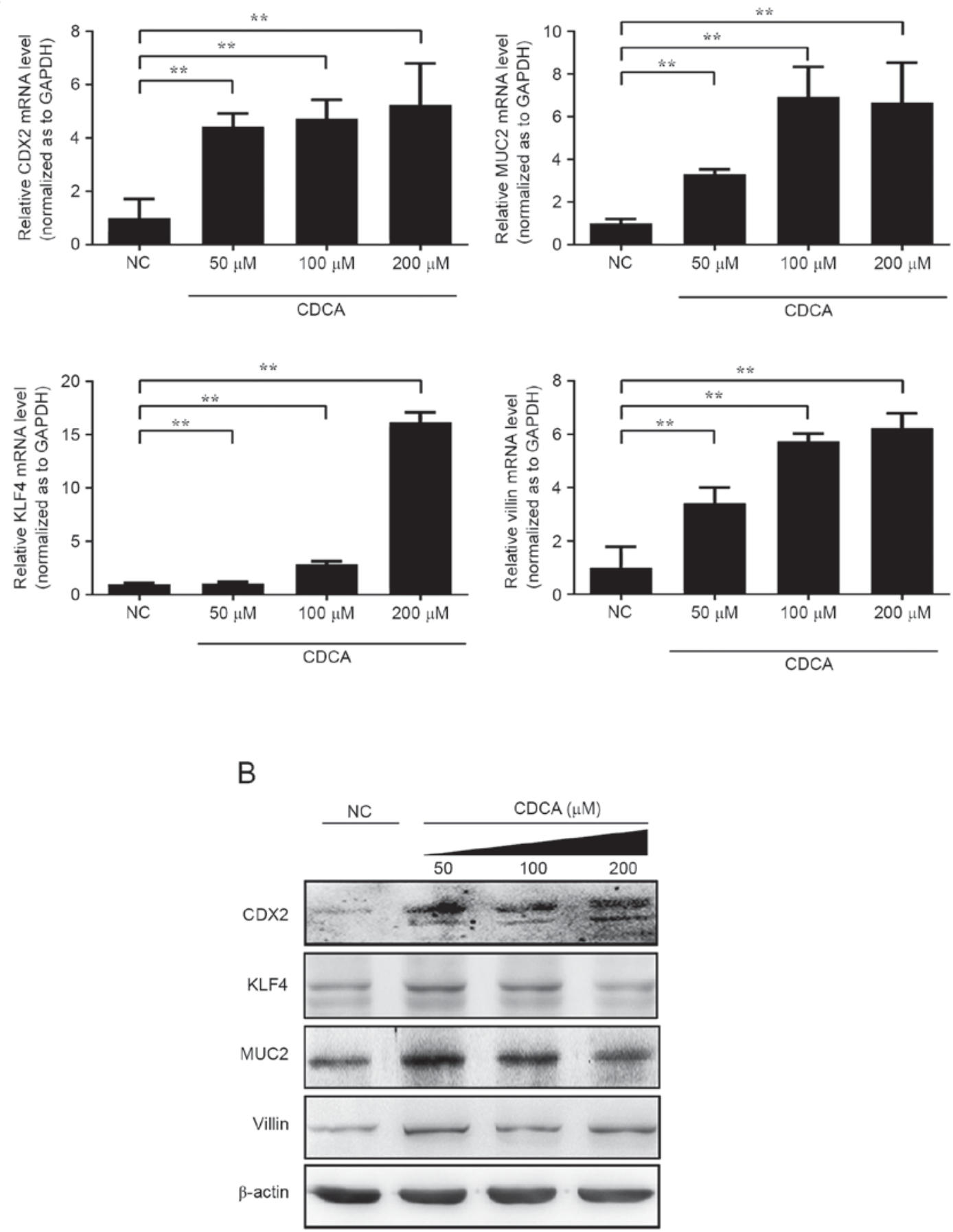

Figure 1. Expression of CDX2, MUC2, KLF4 and villin-1 following treatment with CDCA. CDCA induced the expression of CDX2 and target genes in GES-1 cells. (A) mRNA expression levels of CDX2, MUC2, KLF4 and villin-1 were detected via reverse transcription-quantitative polymerase chain reaction analysis with GAPDH as a control. (B) Protein expression levels of CDX2, MUC2, KLF4 and villin-1 were analyzed using western blot analysis using specific antibodies against CDX2, MUC2, KLF4, villin-1, and $\beta$-actin as a control ( $\left.{ }^{* *} \mathrm{P}<0.01\right)$. CDX2, caudal-related homeobox 2; MUC2, mucin 2; KLF4, kruppel like factor 4; CDCA; chenodeoxycholic acid; NC, negative control.

Relative expression levels of CDX2 and FXR in gastritis and $I M$. In order to further investigate whether there is a correlation between FXR and CDX2, the expression levels of FXR and CDX2 were investigated in clinical samples of IM and gastritis tissues. The clinical characteristics of all patients are shown in Table II. The results of the immunohistochemistry showed that the positive rate of FXR nuclear staining was 91.8\% (56/61) in IM tissues, which was significantly higher than the $54.5 \%(30 / 55)$ in the gastritis tissues $(\mathrm{P}<0.01)$. In addition, elevated expression of CDX2 nuclear staining was detected in $88.5 \%$ (54/61) of the IM tissues, compared with 67.2\% (37/55) of gastritis tissues ( $\mathrm{P}<0.01$; Fig. 5A and B). The expression levels of FXR and CDX2 were positively correlated in the gastritis tissues $(\mathrm{R}=0.764, \mathrm{P}<0.001)$ and IM tissues $(\mathrm{R}=0.642, \mathrm{P}<0.001$; Table III). 

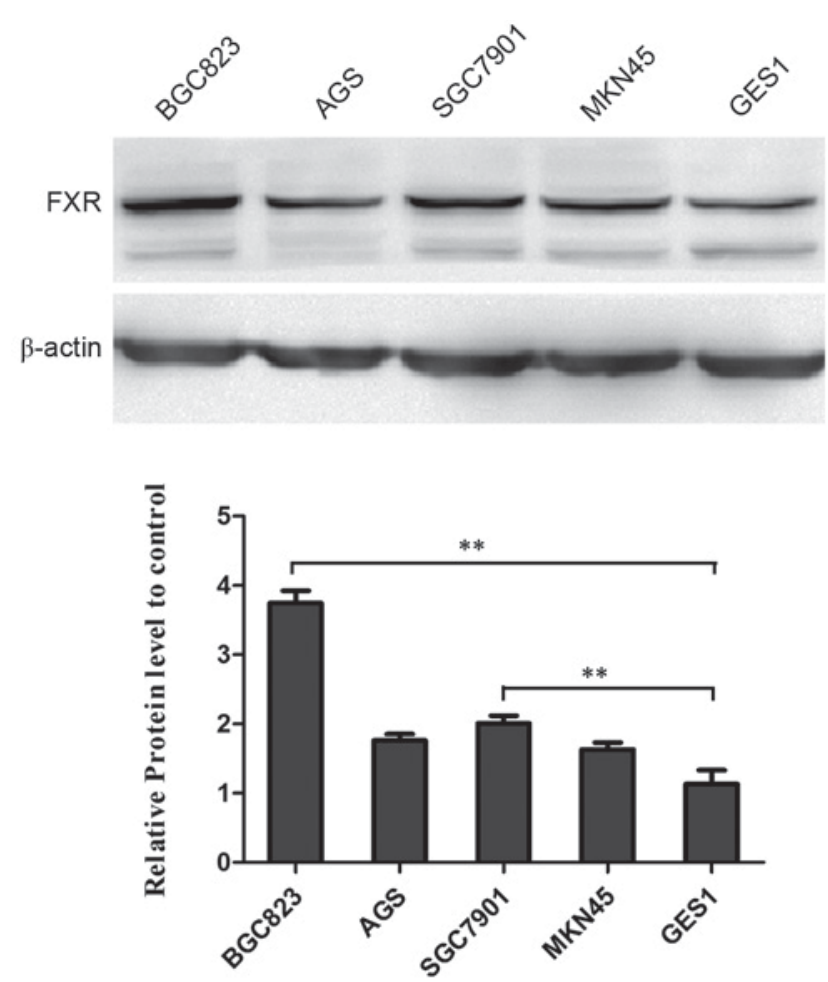

Figure 2. Baseline protein expression levels of FXR in GES-1 normal gastric epithelial cells, BGC-823, AGS, MKN-45 gastric adenocarcinoma cells, and SGC-7901 gastric carcinoma cells. Cell extracts from each sample were separated by sodium dodecyl sulfate-polyacrylamide gel electrophoresis and analyzed using western blot analysis with specific antibodies against FXR and $\beta$-actin as a control. ${ }^{* *} \mathrm{P}<0.01$. FXR, farnesoid $\mathrm{X}$ receptor.

\section{Discussion}

Previous studies using cell lines have showed that the activation of FXR may be involved in the induction of the expression of CDX2 and IM $(6,7)$. However, the association between the expression of FXR and CDX2 in vivo and the exact molecule mechanisms remain to be fully elucidated. In the present study, the in vivo results showed that FXR and CDX2 were concomitantly overexpressed and were positively correlated in IM tissues. In addition, the extensive in vitro results indicated that the upregulation of CDX2 by FXR was dependent on the direct transcriptional induction of SHP.

It has been shown that the reflux of bile acid in the stomach is the most important risk factor for the development of IM in addition to Helicobacter pylori (H. pylori) infection (12). Clinical studies have showed that high concentrations of bile acid appear to be associated with an elevated risk of IM regardless of $H$.pylori infection $(13,14)$. However, the exact underlying molecular mechanisms remain to to be fully elucidated.

IM is characterized by the occurrence of goblet cells, absorptive cells and Paneth cells within the gastric epithelial mucosa. CDX2 is reported to be a direct transcriptional activator of several intestine-specific genes involved in this phenotype, including MUC2, villin-1, intestinal fatty acid-binding protein, glucagon and guanylyl cyclase. Mutoh et al directly showed that $\mathrm{CDX} 2$ contributed to the generation of IM in transgenic mice (15). In addition, several studies using primary cultured esophageal epithelial cells and gastric epithelial cell lines have shown that CDX2 is associated with bile acid-induced cell
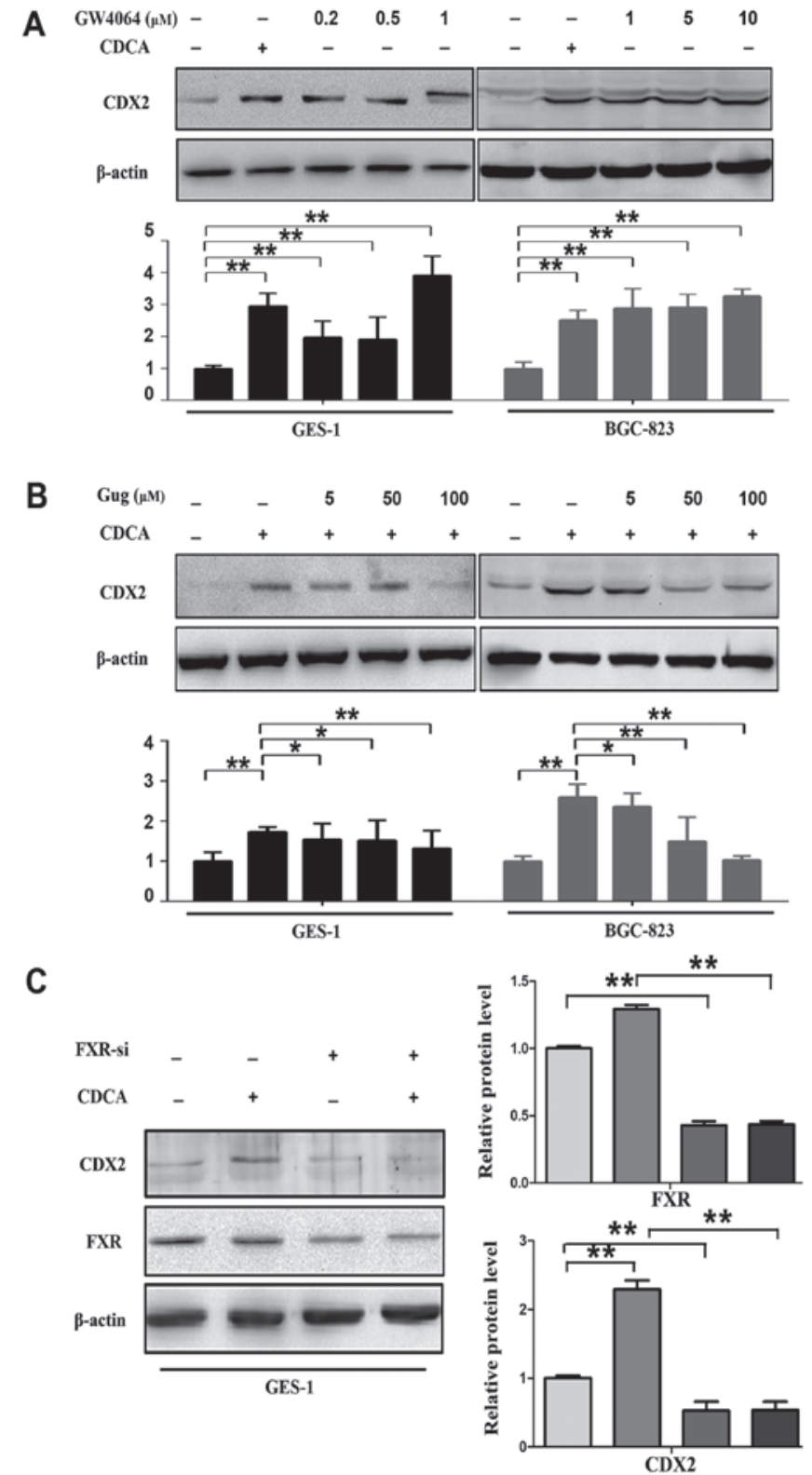

Figure 3. Effects of FXR on CDCA-induced expression of CDX2. Cell extracts from each sample were separated by sodium dodecyl sulfate-polyacrylamide gel electrophoresis and analyzed using western blot analysis with specific antibodies against FXR, CDX2 and $\beta$-actin as a control. (A) CDCA (200 $\mu \mathrm{mol} / 1)$ and GW4064 (1,5 and $10 \mu \mathrm{mol} / \mathrm{l})$ treatment increase the protein expression of CDX2. The cells were exposed for $24 \mathrm{~h}$ with the indicated concentrations of CDCA and GW4064. (B) Protein expression of CDX2 was eliminated by Gug in a dose-dependent manner. Following treatment with CDCA at $200 \mu \mathrm{mol} / 1$, cells were exposed for $24 \mathrm{~h}$ to different concentration of Gug $(5,50$ and $100 \mu \mathrm{mol} / \mathrm{l})$. (C) Protein expression of CDX2 was eliminated by FXR-siRNA. Following $48 \mathrm{~h}$ transfection, the cells were treated for $24 \mathrm{~h}$ with CDCA at $200 \mu \mathrm{mol} / 1 .{ }^{*} \mathrm{P}<0.05,{ }^{* *} \mathrm{P}<0.01$. FXR, farnesoid $\mathrm{X}$ receptor; CDCA, chenodeoxycholic acid; CDX2, caudal-related homeobox 2; siRNA, small interfering RNA; Gug, guggulsterone.

transformation $(16,17)$. In the present study, it was found that CDX2 and target genes were induced by CDCA in normal and gastric cancer cell lines. The results indicated that the induced expression of CDX2 was functional. In support of the in vitro results, the in vivo results indicated that CDX2 was expressed in IM at a significantly higher level, compared with that in gastritis tissues. Taken together, these results revealed that 
A

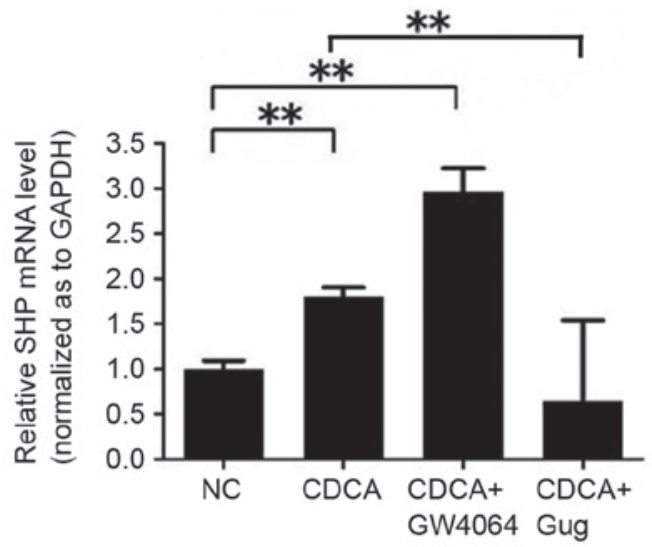

B

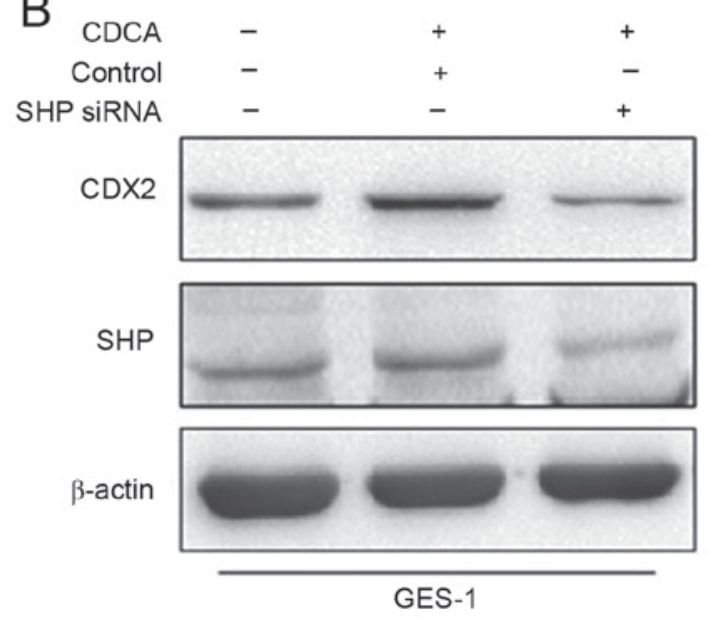

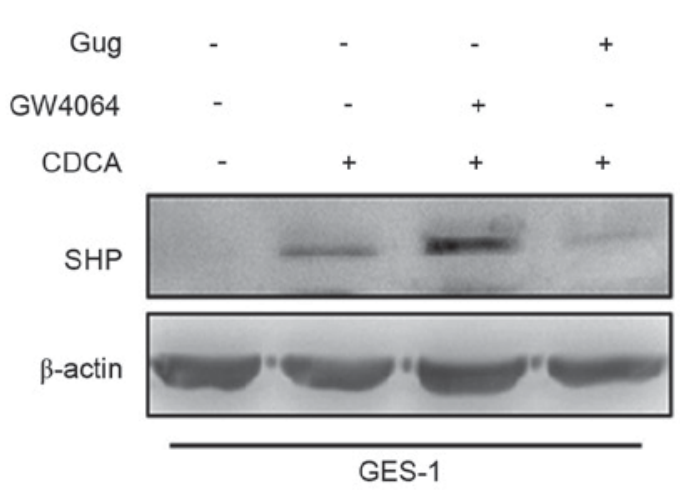

C

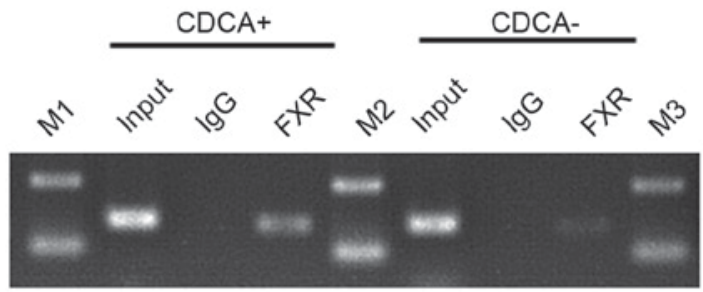

Figure 4. Effect of SHP on the CDCA-induced expression of CDX2. (A) Activation of FXR promoted the expression of SHP at mRNA and protein levels. "* P<0.01. Following treatment with CDCA at $200 \mu \mathrm{mol} / 1$ for $12 \mathrm{~h}$, GES-1 cells were exposed to GW4064 at $1 \mu \mathrm{mol} / 1$ or Gug at $50 \mu \mathrm{mol} / 1$. RT-qPCR analysis was performed following incubation for $12 \mathrm{~h}$ (left) and western blot analysis was performed following incubation for $24 \mathrm{~h}$ (right). (B) Protein expression of CDX2 was eliminated by SHP-siRNA. Following $48 \mathrm{~h}$ transfection, the cells were treated for $24 \mathrm{~h}$ with CDCA at $200 \mu \mathrm{mol} / \mathrm{l}$. (C) ChIP assay. Chromatins were isolated from GES-1 cells following treatment with or without CDCA at $200 \mu \mathrm{mol} / 1$. Binding of FXR to the SHP promoter was examined using the ChIP assay. Following immunoprecipitation with FXR antibody, precipitated DNAs were used for PCR analysis. Rabbit IgG served as control and normal cell lysates were subjected to PCR as input. FXR, farnesoid X receptor; SHP, small heterodimer partner; CDCA, chenodeoxycholic acid; CDX2, caudal-related homeobox 2; Gug, guggulsterone; siRNA, small interfering RNA; RT-qPCR, reverse transcription-quantitative polymerase chain reaction; M1-3, markers 1-3; NC, negative control.

the increased expression of CDX2 was an early event, which occurred prior to the development of IM.

The present study also investigated the upstream signals involved in the induction of CDX2 by CDCA. FXR, acting as a nuclear bile acid sensor, predominantly regulates the metabolism of intracellular bile acid. Previously, the expression of FXR has been reported to be associated with gastric cancer $(18,19)$. In the present study, baseline expression of FXR was observed in normal epithelial and gastric cancer cell lines, and the expression level of FXR was higher in the gastric cancer cell lines. These results indicated that the upregulation of FXR was involved in gastric carcinogenesis. The present study also examined the expression level of FXR in IM and gastritis tissues. The results showed that the level of FXR was significantly higher in IM, compared with that in gastritis, and was positively correlated with the level of CDX2. In addition, GW4064 further promoted the expression of FXR and CDX2. By contrast, following inhibition of the expression of FXR, the expression of CDX2 was almost eliminated. These observations showed that the activation of
FXR by CDCA was involved in the induction of the expression of CDX2.

However, the role of FXR in the gastrointestinal system remains controversial. It has been reported that activation of the FXR pathway protects the gastric mucosa against inflammation-mediated damage $(20,21)$. These findings suggest that the appropriate stimulation of FXR can serve as a protective mechanism. By contrast, other studies have suggested that FXR has a pro-inflammatory role $(21,22)$. The reasons for these contradicting results are unclear, however, they may be associated with the differences in ligands and the genetic disparity of cell lines. Further tissue and cell-specific manipulation of FXR in vivo may elucidate the underlying mechanisms.

The activation of epidermal growth factor receptors (EGFRs) and TGR5 is also involved in gastric intestinal carcinogenesis. Previous reports have suggested that bile acid activates EGFR and TGR5 in the AGS cell line $(23,24)$. In addition, bile acid activates EGFR in other cells lines, including colon cancer cells, cholangiocyte cells and 
Table II. Clinical characteristics of patients.

\begin{tabular}{lccc}
\hline Sample & $\mathrm{n}$ & Male/female & Mean age (years) \\
\hline Gastritis & 55 & $33 / 22$ & 48.5 \\
Intestinal metaplasia & 61 & $42 / 19$ & 55.1
\end{tabular}
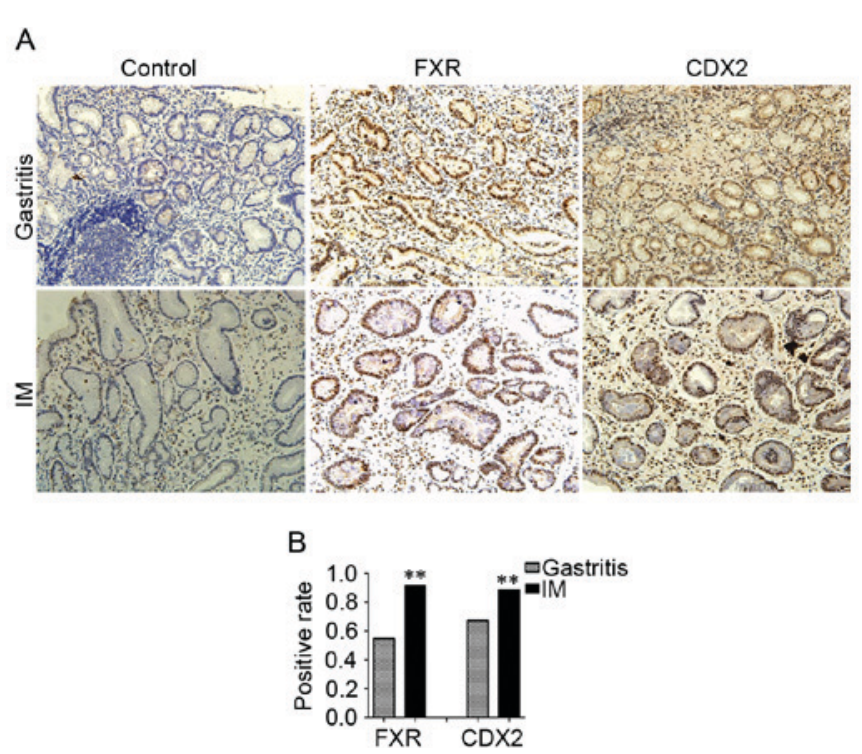

Figure 5. Expression of FXR and CDX2 in IM and gastritis tissues. (A) Tissues were incubated with CDX2 and FXR antibody. Negative controls were incubated with rabbit IgG (magnification, x200). (B) Histological scores were determined using the following formula: Overall score=percentage score $\mathrm{x}$ intensity score. An overall score of $0-12$ was calculated and assigned a negative (score: $0-2$ ) or positive (score: $3-12$ ) grade ("* $\mathrm{P}<0.01$ vs. gastritis). FXR, farnesoid $\mathrm{X}$ receptor; CDX2, caudal-related homeobox 2; IM, intestinal metaplasia.

esophageal adenocarcinoma cells $(25,26)$. Whether the activation of EGFR and TGR5 is involved in the regulation of CDX2 requires further investigation.

SHP is a unique member of the nuclear receptor downstream of FXR, which is important in metabolism and cancer. Previous studies have shown that FXR regulates the expression of SHP through directly binding to an LRH-1 binding site (27). In addition, Park et al demonstrated that the increased expression of SHP is involved in the protein expression of CDX1 in gastric cells induced by bile acid (28). Therefore, it was hypothesized that SHP may also regulate the bile acid-induced expression of CDX2 downstream of the activation of FXR. The results of the present study showed that the activation of FXR enhanced the expression of SHP induced by CDCA, whereas Gug had the opposite effect. In addition, SHP-knockdown eliminated the effects of CDCA on the induced protein expression of CDX2. The results of the ChIP assay showed that CDCA stimulated the DNA binding activity of FXR on the SHP promoter, thus promoting the expression of SHP. However, the molecular mechanisms underlying the effect of SHP on the enhancement of CDX2 remain to be fully elucidated. Previous studies have demonstrated that SHP functionally interacts with NF- $\mathrm{kB}$ as a co-regulator for target gene expression (28). As the CDX2 promoter has two binding sites for NF- $\mathrm{kB}$, the expression of CDX2 may be augmented by the direct interaction of SHP and
Table III. Positive rate of FXR and CDX2 nuclear staining in IM and gastritis tissues.

\begin{tabular}{lccc}
\hline FXR & CDX2+ & CDX2- & P-/R-value \\
\hline Gastritis & & & $<0.001 / 0.764$ \\
+ & 30 & 0 & \\
- & 7 & 18 & $<0.001 / 0.642$ \\
$\mathrm{IM}$ & & & \\
+ & 53 & 3 & \\
- & 1 & 4 & \\
\hline
\end{tabular}

IM, intestinal metaplasia; CDX2, caudal-related homeobox 2; FXR, farnesoid $\mathrm{X}$ receptor.

NF-kB. CDX2 can also bind to its own promoter and induce its expression by an effective auto-regulatory loop (29).

In conclusion, the present study demonstrated in vivo and in vitro that the activation of FXR is involved in the bile acid-induced expression of CDX2 to generate IM. Specifically, the results indicated that bile acid activated the expression of FXR, which then directly unregulated the expression of SHP at the transcriptional level. SHP-knockdown eliminated the effects of CDCA on the induced protein expression of CDX2. Taken together, the observations of the present study suggested that augmentation of the FXR/SHP signal was associated with the phenotypic change of intestinal metaplasia induced by bile acid and gastric carcinogenesis.

\section{Acknowledgements}

Not applicable.

\section{Funding}

The present study was supported by the National Natural Science Foundation of China (grant nos. 81270445, 81370484 and 81470805).

\section{Availability of data and materials}

All datasets generated in the present study are provided in full in the results section of this manuscript.

\section{Author's contributions}

HZ, LZ and YS conceived and designed the study. HZ, TL, and LS performed the experiments. ZN and NL analyzed the results. ZN and $\mathrm{HZ}$ wrote the paper. $\mathrm{ZN}$ and YS reviewed and edited the manuscript. All authors read and approved the manuscript.

\section{Ethics approval and consent to participate}

The study was approved by the Insititutional Review Board of Xijing Hospital. All patients provided written informed consent. 


\section{Consent for publication}

All patients provided written informed consent for the publication of their data and any potentially identifying information was removed.

\section{Competing interests}

The authors declare that they have no competing interests.

\section{References}

1. Correa P, Piazuelo MB and Wilson KT: Pathology of gastric intestinal metaplasia: Clinical implications. Am J Gastroenterol 105: 493-498, 2010

2. Hryniuk A, Grainger S, Savory JG and Lohnes D: Cdx function is required for maintenance of intestinal identity in the adult. Dev Biol 363: 426-437, 2012.

3. Silberg DG, Swain GP, Suh ER and Traber PG: Cdx1 and cdx2 expression during intestinal development. Gastroenterology 119: 961-971, 2000.

4. Xin S, Huixin C, Benchang S, Aiping B, Jinhui W, Xiaoyan L, $\mathrm{Yu} \mathrm{WB}$ and Minhu C: Expression of $\mathrm{Cdx} 2$ and claudin-2 in the multistage tissue of gastric carcinogenesis. Oncology 73: 357-365, 2007.

5. Silberg DG, Sullivan J, Kang E, Swain GP, Moffett J, Sund NJ, Sackett SD and Kaestner KH: Cdx2 ectopic expression induces gastric intestinal metaplasia in transgenic mice. Gastroenterology 122: 689-696, 2002.

6. Li S, Chen X, Zhou L and Wang BM: Farnesoid X receptor signal is involved in deoxycholic acid-induced intestinal metaplasia of normal human gastric epithelial cells. Oncol Rep 34: 2674-2682, 2015.

7. Xu Y, Watanabe T, Tanigawa T, Machida H, Okazaki H, Yamagami H, Watanabe K, Tominaga K, Fujiwara Y, Oshitani N and Arakawa T: Bile acids induce cdx2 expression through the farnesoid $\mathrm{x}$ receptor in gastric epithelial cells. J Clin Biochem Nutr 46: 81-86, 2010.

8. Livak KJ and Schmittgen TD: Analysis of relative gene expression data using real-time quantitative PCR and the 2(-Delta Delta C(T)) method. Methods 25: 402-428, 2001.

9. Matsubara T, Li F and Gonzalez FJ: FXR signaling in the enterohepatic system. Mol Cell Endocrinol 368: 17-29, 2013.

10. Urizar NL, Liverman AB, Dodds DT, Silva FV, Ordentlich P, Yan Y, Gonzalez FJ, Heyman RA, Mangelsdorf DJ and Moore DD: A natural product that lowers cholesterol as an antagonist ligand for FXR. Science 296: 1703-1706, 2002.

11. Park MJ, Kim KH, Kim HY, Kim K and Cheong J: Bile acid induces expression of COX-2 through the homeodomain transcription factor $\mathrm{CDX} 1$ and orphan nuclear receptor SHP in human gastric cancer cells. Carcinogenesis 29: 2385-2393, 2008.

12. Tatsugami $\mathbf{M}$, Ito $\mathbf{M}$, Tanaka $\mathrm{S}$, Yoshihara $\mathbf{M}$, Matsui $\mathrm{H}$, Haruma $\mathrm{K}$ and Chayama K: Bile acid promotes intestinal metaplasia and gastric carcinogenesis. Cancer Epidemiol Biomarkers Prev 21: 2101-2107, 2012.

13. Matsuhisa T and Tsukui T: Relation between reflux of bile acids into the stomach and gastric mucosal atrophy, intestinal metaplasia in biopsy specimens. J Clin Biochem Nutr 50: 217-221, 2012.

14. Matsuhisa T, Arakawa T, Watanabe T, Tokutomi T, Sakurai K, Okamura S, Chono S, Kamada T, Sugiyama A, Fujimura Y, et al: Relation between bile acid reflux into the stomach and the risk of atrophic gastritis and intestinal metaplasia: A multicenter study of 2283 cases. Dig Endosc 25: 519-525, 2013.
15. Mutoh H, Hayakawa H, Sakamoto H, Sashikawa $M$ and Sugano K: Transgenic Cdx2 induces endogenous Cdx1 in intestinal metaplasia of Cdx2-transgenic mouse stomach. FEBS J 276: 5821-5831, 2009.

16. Cooke G, Blanco-Fernandez A and Seery JP: The effect of retinoic acid and deoxycholic acid on the differentiation of primary human esophageal keratinocytes. Dig Dis Sci 53: 2851-2857, 2008.

17. Camilo V, Barros R, Sousa S, Magalhães AM, Lopes T, Mário Santos A, Pereira T, Figueiredo C, David L and Almeida R: Helicobacter pylori and the BMP pathway regulate CDX2 and SOX2 expression in gastric cells. Carcinogenesis 33: 1985-1992, 2012.

18. Duan JH and Fang L: MicroRNA-92 promotes gastric cancer cell proliferation and invasion through targeting FXR. Tumour Biol 35: 11013-11019, 2014.

19. Gadaleta RM, Cariello M, Sabba C and Moschetta A: Tissue-specific actions of FXR in metabolism and cancer. Biochim Biophys Acta 1851: 30-39, 2015.

20. Lian F, Xing X, Yuan G, Schäfer C, Rauser S, Walch A, Röcken C, Ebeling M, Wright MB, Schmid RM, et al: Farnesoid X receptor protects human and murine gastric epithelial cells against inflammation-induced damage. Biochem J 438: 315-323, 2011.

21. Fiorucci S, Mencarelli A, Cipriani S, Renga B, Palladino G, Santucci L and Distrutti E: Activation of the farnesoid-X receptor protects against gastrointestinal injury caused by non-steroidal anti-inflammatory drugs in mice. Br J Pharmacol 164: 1929-1938, 2011.

22. Capello A, Moons LM, Van de Winkel A, Siersema PD, van Dekken H, Kuipers EJ and Kusters JG: Bile acid-stimulated expression of the farnesoid $\mathrm{X}$ receptor enhances the immune response in Barrett esophagus. Am J Gastroenterol 103: 1510-1516, 2008

23. Yasuda $H$, Hirata $S$, Inoue $K$, Mashima $H$, Ohnishi $H$ and Yoshiba M: Involvement of membrane-type bile acid receptor M-BAR/TGR5 in bile acid-induced activation of epidermal growth factor receptor and mitogen-activated protein kinases in gastric carcinoma cells. Biochem Biophys Res Commun 354: 154-159, 2007.

24. Cao W, Tian W, Hong J, Li D, Tavares R, Noble L, Moss SF and Resnick MB: Expression of bile acid receptor TGR5 in gastric adenocarcinoma. Am J Physiol Gastrointest Liver Physiol 304: G322-G327, 2013.

25. Centuori SM, Gomes CJ, Trujillo J, Borg J, Brownlee J, Putnam CW and Martinez JD: Deoxycholic acid mediates non-canonical EGFR-MAPK activation through the induction of calcium signaling in colon cancer cells. Biochim Biophys Acta 1861: 663-670, 2016.

26. Werneburg NW, Yoon JH, Higuchi $\mathrm{H}$ and Gores GJ: Bile acids activate EGF receptor via a TGF-alpha-dependent mechanism in human cholangiocyte cell lines. Am J Physiol Gastrointest Liver Physiol 285: G31-G36, 2003.

27. Hoeke MO, Heegsma J, Hoekstra M, Moshage H and Faber KN: Human FXR regulates SHP expression through direct binding to an LRH-1 binding site, independent of an IR-1 and LRH-1. PLoS One 9: e88011, 2014.

28. Park WI, Park MJ, An JK, Choi YH, Kim HY, Cheong J and Yang US: Bile acid regulates c-Jun expression through the orphan nuclear receptor SHP induction in gastric cells. Biochem Biophys Res Commun 369: 437-443, 2008.

29. Barros R, da Costa LT, Pinto-de-Sousa J, Duluc I, Freund JN David L and Almeida R: CDX2 autoregulation in human intestinal metaplasia of the stomach: Impact on the stability of the phenotype. Gut 60: 290-298, 2011.

This work is licensed under a Creative Commons Attribution-NonCommercial-NoDerivatives 4.0 International (CC BY-NC-ND 4.0) License. 